\title{
Dynamic Cardiac Mapping on Patient-Specific Cardiac Models
}

\author{
Kevin Wilson ${ }^{1,3}$, Gerard Guiraudon ${ }^{1,2}$, Doug Jones ${ }^{1,2,4}$, Cristian A. Linte ${ }^{1,3}$, \\ Chris Wedlake $^{1}$, John Moore ${ }^{1}$, and Terry M. Peters ${ }^{1,3,5}$ \\ ${ }^{1}$ Imaging Research Laboratories, Robarts Research Institute, \\ ${ }^{2}$ Canadian Surgical Technology and Advanced Robotics (CSTAR), \\ ${ }^{3}$ Biomedical Engineering Graduate Program, University of Western Ontario, \\ ${ }^{4}$ Department of Medicine, University of Western Ontario, \\ ${ }^{5}$ Department of Medical Biophysics, University of Western Ontario, \\ London, Ontario, Canada \\ kevin.wilson@vanderbilt.edu, tpeters@imaging.robarts.ca
}

\begin{abstract}
Minimally invasive techniques for electrophysiological cardiac data mapping and catheter ablation therapy have been driven through advancements in computer-aided technologies, including magnetic tracking systems, and virtual and augmented-reality environments. The objective of this work is to extend current cardiac mapping techniques to collect and display data in the temporal domain, while mapping on patient-specific cardiac models. This paper details novel approaches to collecting spatially tracked cardiac electrograms, registering the data with a patient-specific cardiac model, and interpreting the data directly on the model surface, with the goal of giving a more comprehensive cardiac mapping system in comparison to current systems. To validate the system, laboratory studies were conducted to assess the accuracy of navigating to both physical and virtual landmarks. Subsequent to the laboratory studies, an in-vivo porcine experiment was conducted to assess the systems overall ability to collect spatial tracked electrophysiological data, and map directly onto a cardiac model. The results from these experiments show the new dynamic cardiac mapping system was able to maintain high accuracy of locating physical and virtual landmarks, while creating a dynamic cardiac map displayed on a dynamic cardiac surface model.
\end{abstract}

\section{Introduction}

Minimally invasive cardiac therapy, driven through computer-aided intervention, has made a large contribution to the advancement in the field of cardiac mapping and catheter ablation therapy. Dedicated systems such as the CARTO ${ }^{\mathrm{TM}}$ XP Navigation System (Biosense-Webster), EnSite ${ }^{\circledR}$ System (St. Jude Medical), and more recently the combination of the CARTO RMT electroanatomical mapping system with the Stereotaxis Niobe magnetic steering technology, provide physicians with the tools necessary to record, display, and analyze the electrical activation patterns of the heart. 
The motivation behind cardiac mapping systems for both clinical and research related use are to create a detailed description of the electrical activation of the heart to study the mechanisms of arrhythmias, and in some cases assist in a curative procedure such as catheter ablation therapy. In practice, atrial fibrillation ( $\mathrm{AF}$ ) is the most common type of cardiac arrhythmia affecting over 2.5 million North Americans, and due to an aging population is becoming more prevalent 1. However, researchers are still investigating the sources of AF. As reported by Efimov and Fedorov [2] theories are being investigated whether AF is caused by single or multiple sources, the electrical circuits are focused or reentrant, and whether AF is purely and myogenic disease or whether there are neurological causes.

A key tool in this analysis is a cardiac map, which gives spatial representation to cardiac electrophysiological (EP) data by tracking the tip of the electrode catheter in real-time, while recording cardiac electrograms (CEGs). The tracked location data are reconstructed into a three dimensional model and the CEG data is interpolated across the structure so information such as activation time, signal amplitude, as well as abnormal patterns can be studied on the volumetric atrial/ventricle and epicardial/endocardial surfaces.

Until recently cardiac mapping systems were limited to maps displayed on reconstructed heart geometry created through the sampled locations collected from the tracked catheter. This limited the usefulness of the map to the ability of the physician to orient the data with its corresponding anatomical locations. Now, the CARTO ${ }^{\mathrm{TM}}$ Merge and EnSite ${ }^{\circledR}$ Verismo modules provide tools for importing pre-operative patient data, segmenting heart geometry to create virtual models, and overlaying the models on the reconstructed cardiac map. This provides valuable information to the physician by allowing them to more easily associate the collected EP data with known anatomical locations.

The purpose of this work is to extend the current approaches in cardiac mapping to create a system that more accurately represents the collected information, allows physicians additional tools to interpret the data, and provides a more robust cardiac surgery platform to deliver therapy. We propose to do this by describing and evaluating a dynamic cardiac mapping system, using patientspecific cardiac models. This system extends the collection, analysis, and display of the spatially tracked CEGs into the temporal domain, while providing the ability for the data to be displayed directly on a patient-specific model created from pre-operative cardiac images. In addition, the cardiac mapping system is to be included in the our virtual-reality based cardiac surgery and therapy environment that supports integrated modular components 34, to provide a more robust platform for delivering cardiac care.

\section{Method}

The hardware components of our dynamic cardiac mapping system consist of a $3.0 \mathrm{GHz}$ Pentium 4 computer workstation that serves as the central core. The 
workstation processes data from an Aurora magnetic tracking system (MTS) (Northern Digital Inc., Waterloo, Canada). The MTS sensors are rigidly attached to a standard electrode catheter for the purpose of tracking the location and orientation of the tip of the catheter. The electrode is connected to an analogto-digital converter (ADC) (Data Translation, Marlboro, USA) for input to the workstation that also records the patient's digitized ECG signal. The workstation is based on running the Atamai Viewer software package, which is responsible for processing all the data. The Atamai Viewer cardiac surgery planning and therapy environment supports integrated module components including image viewing, volume rendering, ultrasound overlay, surgical tracking, as well as the dynamic EP cardiac mapping environment described in this work.

\subsection{Pre-operative and Configuration}

A dynamic structural cardiac scan (CT or MR) of the patient is taken in a preoperative stage of the cardiac mapping procedure. This image is segmented to create a patient-specific dynamic cardiac model that will be used in our system as the geometric structure for displaying the cardiac map. The most simplistic approach to the segmentation is manually outlining the region(s) of interest in each image, and combining each outline to create a polygonal surface. More advanced software methods and programs for segmentation exist, examples in the cardiac mapping field are CARTO ${ }^{\mathrm{TM}}$ Merge and EnSite ${ }^{\circledR}$ Verismo. For our system, we have used the method described by Wierzbicki et al. [5], which uses non-linear registration techniques to propagate a segmented surface from one cardiac phase to another in order to generate a dynamic cardiac model. The cardiac model is imported into the Atamai Viewer and the display is synchronized to the patient's heart rhythm using the ECG signal. Throughout the entire procedure the synchronization between the patient's heart pace and the model pace is constantly updated.

Following the synchronization of the display, a two-step procedure is completed to characterize the electrode catheter and the MTS. This step is necessary to transform the locations reported by the MTS relative to the field generator to locations relative to anatomical space. The first procedure is to find the transformation for the location of the sensor in respect to the catheter tip, and is achieved through a pivot calibration algorithm that finds a transformation to minimize the RMS distance between the sensor's location and a pivot point while posing the tracked catheter in different positions, keeping the tip on the pivot point. The second transformation is found to transform points from the MTS coordinate system to the cardiac model's coordinate system. Initially, this transformation is found through a landmark-based registration, to orient the coordinate systems and approximately align the cardiac model with the patient's heart. After the initial transformation is achieved, a fully automatic temporal based point registration as described previously [6], refines the alignment of the physical and virtual environments.

Once the patient-specific cardiac model is created, and the spatial locations reported by the MTS are transformed to represent known physical locations, 
the dynamic cardiac mapping system is prepared to collect data and create the cardiac map.

\subsection{Data Collection}

For each sampled location the system collects a dynamic set of spatial coordinates reported by the MTS, representing the locations at which the CEG is sampled. Associated with these locations are the CEG samples from the electrode catheter, which the system records through the ADC.

To sample a location the physician holds the catheter against the moving cardiac chamber wall, presses the acquisition button, and holds the catheter at the same location on the beating heart until the system indicates the acquisition is complete. From a systems point-of-view, once the acquisition is fired the system waits for the next R-wave trigger from the ECG signal, and begins to sample spatial locations and the CEG signal for an entire cardiac cycle. Temporal alignment occurs after data collection, as the timing of each device is derived from a single system clock, and the timestamps associated with each signal.

The data are stored within the system and are available to the physician to analyze in both time and frequency domains. In addition, to viewing the data in association with the patient-specific cardiac model, the data are grouped into separate point-cloud sets. The imported dynamic cardiac model has a finite number of surfaces that each represents a different phase of the cardiac model (10 surfaces). Through the time course of the collected data the values of the spatial coordinate and the CEG are extracted at each of these phases and inserted into a corresponding point cloud. The result of this procedure is 10 separate point cloud datasets where the location of each point is the physical location of the CEG along with the recorded value. Throughout the cardiac mapping procedure, additional locations are sampled over the myocardium and the point clouds become denser, leading to a more accurate cardiac map.

\subsection{Surface Reconstruction and Interpolation}

Once four or more locations have been collected the data may be constructed into a dynamic surface model. Similar to current cardiac mapping systems, this system allows users to reconstruct cardiac anatomy based on collected spatial locations. The points within a point cloud are connected using a Delaunay triangulation, and the resulting mesh is input into a geometry filter that extracts tetrahedral facets on the outer surface of the mesh, representing the geometry of the cardiac chamber. Data values from the CEG at each collected location are linearly interpreted onto the surface, and users are able the view the cardiac data as a color coded map, represented as either peak voltage or relative activation time.

The final step in the creation of a dynamic cardiac map is to interpolate the values from the raw data onto the patient-specific cardiac model. In the pre-operative stage the model was created from a dynamic CT or MR image, and registration between the physical and virtual environment was achieved with an automatic temporal based algorithm. Since the raw data and model 
are in the same coordinate system we can choose a radius of interpolation, $\mathrm{Ri}$, which determines the distance around each raw data point that will receive an interpolated CEG value on the cardiac map. Once the areas to be interpolated are determined, the closest point on the patient-specific cardiac model to each point on the reconstructed raw dynamic cardiac map is calculated and assigned a value, completing the creation of the dynamic patient-specific cardiac model.

\section{Experiments}

Three separate experimental protocols were set up to evaluate the overall effectiveness of the dynamic cardiac mapping system. The experiments assessed the accuracy of locating a physical landmark, the accuracy of locating a landmark in image space, and the creation of a dynamic cardiac map in an in-vivo porcine experiment.

\subsection{Physical Accuracy and Image Space Accuracy Experiments}

Methodology. For the accuracy experiments we employed a realistic beating heart phantom (The Chamberlain Group, Great Barrington, MA, www.thecgroup.com) as the cardiac model. Five CT visible fiducial markers were fixed to the surface of the phantom, and a dynamic 10-phase CT image volume was acquired. These data were then used to create a dynamic cardiac surface model.

In both the physical and image space accuracy studies a dynamic experiment was conducted to represent our cardiac mapping environment, and a static experiment was performed to mimic current cardiac mapping system technology.

To measure physical and image space accuracy in the static environment, one phase of the cardiac model was imported into the system, and configured as described in the pre-operative stage above. To simulate a cardiac mapping procedure using the current technology, 20 locations were sampled around the epicardium of the phantom and an iterative closest point (ICP) registration was performed 7]. Seven users were asked to select the five fiducial markers using a tracked catheter while directly looking at the phantom for the physical accuracy study, and while looking only at the computer display (virtual environment) for the image space accuracy study. Each user repeated the physical study three times and the virtual study twice. The values were then compared with the ground truth, positions identified directly on the CT image.

Next, the cardiac mapping system was configured for dynamic mapping by importing all 10 phases of the cardiac model. The system was configured and 20 locations were sampled around the epicardium, however in this experiment the temporal based registration methods were used to align the coordinate systems of real space with image space. The same users were asked to repeat the same procedure as in the static environment. There were two key differences in this experiment; the system acquires multiple coordinates at each sampled location (corresponding to the movement of the cardiac wall throughout each phase), and in the virtual environment the users follow a dynamic target on the screen. 
Results. The following errors are important in the evaluation of the systems. The fiducial localization error (FLE), measured as a RMS distance error, is defined by the NDI Aurora MTS system specifications as $0.9 \mathrm{~mm}$ for translation and $0.8^{\circ}$ for orientation. The target registration error (TRE) of the catheter tip was $1.54 \mathrm{~mm}$ measured using the pivot calibration algorithm. These errors have a direct effect on the overall accuracy of the system.

The targeting accuracy was reported as the RMS distance from the recorded coordinates of the above experiments with the ground truth location of the given fiducial. Table 1 summarizes these errors for the dynamic and static mapping systems for each the physical and image space accuracies. Furthermore, a quantile-to-quantile (q-q) plot was created for each result set, comparing the quantiles of the error distribution against a normal distribution, with a linear result confirming a normal distribution of the errors.

Table 1. Accuracy measurements of the cardiac mapping system

\begin{tabular}{lcc} 
Experiment & System & RMS Error \\
\hline Physical Space Accuracy & Static & $3.7 \mathrm{~mm}$ \\
& Dynamic & $3.9 \mathrm{~mm}$ \\
Image Space Accuracy & Static & $3.7 \mathrm{~mm}$ \\
& Dynamic & $3.7 \mathrm{~mm}$
\end{tabular}

Student's t-test analysis demonstrated no significant difference between the two cases in each experiment, and a one-way ANOVA test showed no significant differences between the performances of the various volunteers.

The results demonstrate that we are able to extend the current technology into a dynamic environment while maintaining the accuracy of locating landmarks. In context of catheter ablation therapy, these maintained accuracies are within our goal of $4 \mathrm{~mm}$, in order to create a fully connected isolation ring around an AF focal region using a standard $8 \mathrm{~mm}$ ablation catheter in a clinical environment.

\subsection{In-Vivo Porcine Experiment}

Methodology. We evaluated this system clinically in an in-vivo porcine study. To create a gold standard comparison, a custom-made 63-electrode plaque (electrodes spaced at $4 \mathrm{~mm}$ ) was sutured on to the right atrium (RA) of a pig. The recordings from the electrode were input to a Prucka CardioLab EP System (GE Healthcare). Through this system, a 2D isochronal map of activation time can be created. Once the plaque was in place a quadripolar electrode was sutured on the RA superior to the plaque, and was used to pace the pig's heart at a rate of $1.9 \mathrm{~Hz}$.

The in-vivo experiment was conducted on the porcine heart exposed via a mini-thoracotomy as part of a separate porcine electrophysiology study. A CT-derived porcine dynamic heart model was imported in the Atamai Viewer software package, within which two dynamic cardiac maps were created. The first was a six-point dynamic isochronal cardiac activation map generated while 

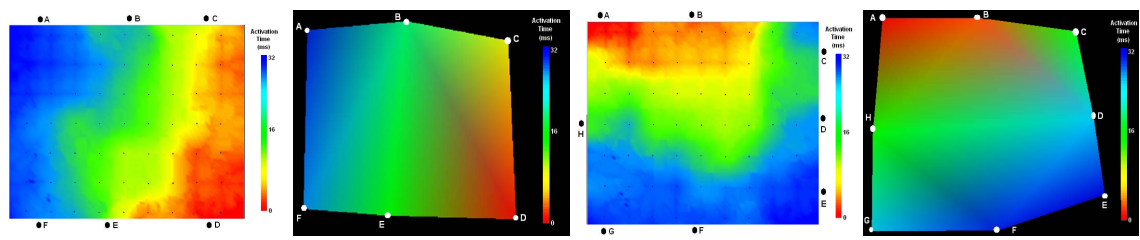

Fig. 1. Cardiac maps of the RA of a pig heart. From left to right: 63-point 2D isochronal map during pacing (Prucka System), 6-point 3D isochronal map during pacing (Dynamic System), 63-point 2D isochronal map during sinus rhythm (Prucka System), 8-point 3D isochronal map during sinus rhythm (Dynamic System).

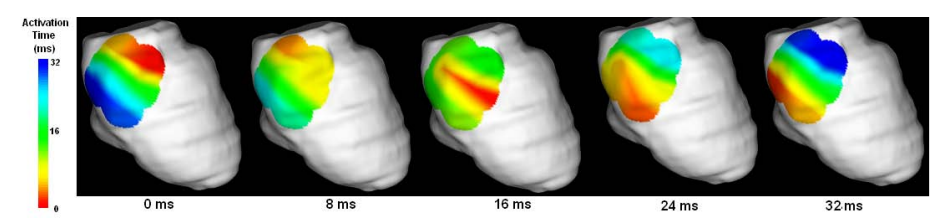

Fig. 2. Dynamic series of 6-point isochronal cardiac activation maps interpolated on a cardiac surface model created during pacing of the heart

pacing the heart, by sampling at each corner and one on either side at the middle of the plaque. The second was an eight-point map was created during sinus rhythm from sampling around the plaque (each corner and the middle of each edge).

Results. Figure 1 shows the cardiac maps created for each system during paced and sinus rhythm of the heart. On each pair of maps (paced/sinus for each system) the sampled location was marked to indicate overlapping portions of the map. All images are color coded, indicating reference activation time (red) to activation $32 \mathrm{~ms}$ later (blue).

From these images it can be seen that the activation patterns from the dynamic mapping system match well with those from the Prucka system. In the paced recording the activation is starting from the lower-right corner, where the quadripolar electrode was placed, and proceeding across the plaque area from right to left. During sinus rhythm the pacing initiates in the top-middle-left of the plaque area, where the sinoatrial (SA) node is closely located, and proceeds across the RA toward the bottom of the plaque area. These results demonstrate the system's ability to record CEG data in conjunction with their spatial location and reconstruct a meaningful dynamic cardiac map on a pre-operative CT model (Figure 2).

\section{Conclusion}

In this work we have introduced methods to extend the current cardiac mapping technologies to collect dynamic cardiac electrophysiology data and display the 
generated cardiac map directly on a patient-specific model. By recording CEGs and the motion of the cardiac wall over the entire cardiac cycle we are able to temporally align the data and create discrete point cloud datasets corresponding to different phases of the cardiac cycle. These datasets also correspond to a dynamic cardiac surface model that has been pre-operatively created from a $4 \mathrm{D}$ CT or MR image. The system registers coordinate systems between the tracking system and the imaging space, allowing users the ability to interpret the data onto the patient-specific model.

We have shown the ability to extend current cardiac mapping techniques to the dynamic patient-specific system without losing accuracy of locating physical landmarks or the ability to navigate using a dynamic virtual environment. In the in-vivo study we demonstrated that the system is able to collect and temporally align data to reproduce cardiac maps created using a commercial EP system. However, the system extends the usability of current technologies by allowing physicians to more freely analyze the entire CEG in context to the patient's anatomy throughout different phases of the cardiac cycle.

\section{References}

1. Go, A.S., Hylek, E.M., Phillips, K.A., Chang, Y., Henault, L.E., Selby, J.V., Singer, D.E.: Prevalence of diagnosed atrial fibrillation in adults: national implications for rhythm management and stroke prevention: the AnTicoagulation and Risk Factors in Atrial Fibrillation (ATRIA) Study. JAMA 285(18), 2370-2375 (2001)

2. Efimov, I.R., Fedorov, V.V.: Chessboard of atrial fibrillation: reentry or focus? Single or multiple source(s)? Neurogenic or myogenic? American Journal of Phyiology Heart and Circulatory Physiology 289, H977-H979 (2005)

3. Moore, J., Guiraudon, G., Jones, D., Hill, N., Wiles, A., Bainbridge, D., Wedlake, C., Peters, T.: 2D ultrasound augmented by virtual tools for guidance of interventional procedures. Health Technology and Informatics 125, 322-327 (2007)

4. Linte, C.A., Wiles, A.D., Moore, J., Wedlake, C., Peters, T.M.: Virtual realityenhanced ultrasound guidance for atrial ablation: In vitro epicardial study. In: Metaxas, D., et al. (eds.) MICCAI 2008, Part II. LNCS, vol. 5242, pp. 644-651. Springer, Heidelberg (2008)

5. Wierzbicki, M., Drangova, M., Guiraudon, G., Peters, T.: Validation of dynamic heart models obtained using non-linear registration for virtual reality training, planning, and guidance of minimally invasive cardiac surgeries. Medical Image Analysis 8(3), 387-401 (2004)

6. Wilson, K., Guiraudon, G., Jones, D., Peters, T.M.: 4D shape registration for dynamic electrophysiological cardiac mapping. In: Larsen, R., Nielsen, M., Sporring, J. (eds.) MICCAI 2006. LNCS, vol. 4191, pp. 520-527. Springer, Heidelberg (2006)

7. Besl, P.J., McKay, N.D.: A method for registration of 3-D shapes. IEEE Transactions on Pattern Analysis and Machine Intelligence 14(2), 239-256 (1992) 